YVANKA B. RAYNOVA (Sofia/Wien)

\title{
Über-Setzung als diskursive Dominanz: Paul Ricœurs Übersetzungsparadigma neu gelesen
}

\author{
Über-Setzung as a discursive Dominance: \\ A new Interpretation of Paul Ricoeur's Paradigm of Translation
}

\begin{abstract}
Putting into question the idealized interpretations of translation as an ethical paradigm and model for Europe (Paul Ricoeur, Domenico Jervolino), the author aims to reveal some negative aspects related to the politics of translation, specially the use of translation as a transfer of a dominant discourse. This negative phenomenon is analyzed by mean of the neologism of Über-Setzung (superimposition) designating an overlap of a discourse or an idiom over another through the abuse of the linguistic hospitality of translation. On the base of diverse examples and narratives the author offers a description of the eidetic structure of Über-Setzung, and argues that the dominating language and its discourse intervene in the mother language and its culture by putting itself "on" and "over" them as something primary, absolute and universal. The dominant discourse, that is established by means of translation, requires mindfulness, comprehension and acceptance; however, because it is a kind of anti-giving, the dominator who use it refuses to recognize the dominated as a partner; he treats him as non-equal and even as "retarded". In conclusion the author displays the intercultural potentialities of hermeneutic phenomenology in regard of a new European ethos of translation.
\end{abstract}

Keywords: Paul Ricœur, translation, hermeneutics, ethics, hospitality, domination, European integration

In seinem Artikel "Le paradigme de la traduction" (1998)1 verweist Paul Ricœur auf den Babel-Mythos als dem Ereignis der originären Spaltung der Sprachen, die für ihn zwar irreversibel ist, jedoch keine endgültige Verhinderung der Verständigung darstellt. Die Tatsache der Zerstreuung und der Pluralität der Sprachen wird von ihm zum Anlass

${ }^{1}$ Dieser Artikel wurde erstmals in der Zeitschrift Esprit veröffentlicht (Paul Ricœur, "Le paradigme de la traduction", in Esprit, no. 6, juin, 1998, 8-19) und erschien später mit zwei anderen Beiträgen als Broschüre unter dem Titel Sur la traduction (Ricœur, 2004). 
genommen, ein Plädoyer für die Übersetzung und die sprachliche Gastfreundschaft als ethischen Grundsatz zu formulieren:

Es scheint mir, in der Tat, dass die Übersetzung nicht nur eine intellektuelle, theoretische oder praktische Arbeit, sondern auch ein ethisches Problem darstellt. Den Leser zum Autor zu bringen, den Autor zum Leser, mit dem Risiko zweier Herren zu dienen und sie zu verraten, ist das, was ich so gerne mit sprachliche Gastfreundschaft bezeichne. Sie ist ein Modell für die anderen mit ihr verwandten Formen der Gastfreundschaft: Die Glaubensbekenntnisse, die Religionen, sind sie nicht wie fremde Sprachen unter einander, mit ihrem Sprachschatz, mit ihrer Grammatik, Rhetorik und Stilistik, die man erlernen muss, um in ihnen durchdringen zu können?² (Ricœur 2004, 42-43).

Diese ethische Perspektive der Übersetzung als sprachliche Gastfreundschaft, auf die uns auch Domenico Jervolino in seinem hier, in dieser Nummer, veröffentlichten Beitrag aufmerksam macht (Jervolino 2014), wird bei Ricœur schon einige Jahre früher in seinem wenig bekannten Aufsatz "Quel éthos nouveau pour l'Europe?" entwickelt. (Ricœur 1992) Dieser Text hat meines Erachtens eine besondere Bedeutung, da er die Übersetzung als ein Modell der europäischen Integration auslegt und zwar auf eine sehr originelle Art und Weise. Um die neue, "postnationale" Situation in einem Vereinigten Europa zu erfassen, in der Identität und Differenz dialektisch zu denken sind, stellt Ricœur zwei mögliche Modelle der europäischen Integration vor: Die Übersetzung (la traduction) und den Gedächtnisaustausch (l'échange de mémoires). Die Übersetzung stellt führ ihn deshalb ein angemessenes Modell dar, weil Europa immer mehrsprachig sein wird. Dieses Modell beinhaltet sowohl weitgehende Forderungen als auch Versprechen, die bis ins Herz des ethischen Lebens der Individuen und der Völker reichen. Ricœur ruft hier das Humboldt'sche Modell der Übersetzung in Erinnerung, welches dazu anregt, die eigene Sprache auf das Niveau der Fremdsprache zu bringen, insbesondere wenn es um originelle Errungenschaften geht, die eine Herausforderung für die Aufnahmesprache darstellen. "Es geht darum" - erklärt Ricœur - "beim Anderen zu hausen, damit man ihn zu sich als einen eingeladenen Gast hinführt". (Ricœur 1992, 110) Gerade das sei beim Aufbau der Europäischen Union vonnöten:

${ }^{2}$ Dieses Zitat, sowie alle folgenden aus dem Aufsatz "Quel éthos nouveau pour l'Europe?" sind in meiner Übersetzung aus dem Französischen. 
Auf der Ebene der Institutionen ladet es [das Modell der Übersetzung] dazu ein, im ganzen europäischen Raum den Unterricht zweier lebendiger Sprachen, die sich nicht in einer dominanten Position befinden, zu fördern, um ihnen ein Auditorium zu sichern. Doch insbesondere auf der geistigen Ebene ladet es dazu ein, die Übersetzung auf die interkulturellen Beziehungen auszuweiten, d.h. auf die Sinnesinhalte, die durch die Übersetzung gesteuert werden. Gerade hier werden Übersetzer von Kultur zu Kultur gebraucht, zweisprachige Übersetzer von Kulturen, die in der Lage sind diesen Transfer im mentalen Universum der anderen Kultur durchzuführen, unter Berücksichtigung ihrer eigentümlichen Sitten, fundamentalen und wichtigsten Überzeugungen, also ihrer gesamten Sinnreferenzen. In diesem Sinn kann man von einem Ethos der Übersetzung sprechen, dessen Ziel auf der kulturellen und geistigen Ebene die Geste der sprachlichen Gastfreundschaft wiederholt. (Ricœur 1992, 110)

Das ethische Paradigma der Übersetzung wird von dem Ricœur-Schüler Domenico Jervolino im Sinne einer Gabe verstanden und als Ansatz zu einer neuen europäischen Politik umgedeutet ${ }^{3}$ :

Zuletzt möchte ich darauf hinweisen, dass die Idee von Übersetzung (...), sogar die Politik beeinflussen kann. Fragen wir uns, welche heute die Sprache eines vereinigten Europas sei, das sich in einer globalisierten Welt befindet, die an vielen Orten von Kriegen und von Gewalt erschüttert ist. Nun, darauf antworte ich (...), dass die europäische Sprache die Übersetzung ist. Insbesondere in Übereinstimmung mit Etienne Balibar (...) bin ich der Überzeugung, dass Europa, durch seine jahrhundertlange Geschichte voller Konflikte und Kriege gereift, dazu berufen ist, Übersetzer und Vermittler der Welt zu werden und die Begegnung zwischen den Kulturen, den Religionen und den Nationen mit einer (aktiven) Friedenspolitik zu fördern, vor allem im Mittelmeerraum und auf dem Balkan. (Jervolino 2016, 60)

Ricœur und Jervolino deuten die Übersetzung in einem fast ausschließlich positiven Licht. Beide sehen zwar gewisse Schwierigkeiten in linguistischer und semantischer Hinsicht, die mit der Unmöglichkeit einer perfekten, absolut getreuen Übersetzung von einer Sprache in die andere zusammenhängen. Während Ricœur die ethische und geistige Bedeutung der Übersetzung für die europäische Integration hervorhebt, geht Jervolino jedoch wesentlich weiter, indem er ihr auch einen politischen Einfluss beimisst und in ihr

${ }^{3}$ Jervolino bezieht sich jedoch nur auf die drei Studien von Ricœur, veröffentlicht in der Broschüre Sur la traduction (Ricœur, 2004), ohne Bezug auf den Aufsatz "Quel éthos nouveau pour l'Europe?". 
die Möglichkeiten einer neuen, spezifisch europäischen Konfliktlösungsstrategie bei Kriegen und internationalen Problemen sieht.

Gegen diese extrem positiven und gewissermaßen idealisierenden Interpretationen der Übersetzung, die real existierende Dominanzverhältnisse in der Übersetzungspolitik ignorieren und ausblenden, möchte ich meinerseits die ethischen und politischen Probleme der Übersetzung durch einen phänomenologisch-hermeneutischen Umweg thematisieren, der nicht nur die positiven Aspekte, sondern auch die Schattenseiten zum Vorschein bringt. Für diesen Zweck werde ich den Arbeitsbegriff der "Über-Setzung" einführen als Bezeichnung für eine besondere Form der Herrschaft über und durch die Sprache mittels Ausnutzung der Gabe der sprachlichen Gastfreundschaft der Übersetzung. Der konkrete Gehalt dieses Begriffs und seine allgemeinen Strukturen sollen in der folgenden Untersuchung interkulturell anhand diverser Beispiele und Narrative ausgelegt werden. Ich werde jedoch die hermeneutische Phänomenologie als philosophische Methode hier in einer anderen Reihenfolge anwenden als Ricœur. Ich werde zuerst von der empirischphänomenologischen Beschreibung des Kontextes ausgehen, d.h. von der Übersetzung in einem "osteuropäischen" Kontext und erst danach, auf der Basis gewisser Diskurskonflikte, die eidetische Beschreibung der Über-Setzung" vornehmen. Dies soll gewisse Schlussfolgerungen ermöglichen und zugleich das Potential der hermeneutischen Phänomenologie in Bezug auf einen neuen europäischen Übersetzungsethos aufzeigen.

\section{1. Öffnung und Verschluss}

Zunächst möchte ich auf den positiven Aspekt und den Beitrag hinwiesen, den die Übersetzungsarbeit in den "osteuropäischen" Ländern während der letzten 25 Jahren geleistet hat. Es ist bekannt, dass in diesem Teil Europas vor der Wende das ganze kulturelle Leben, inklusive der literarischen und wissenschaftlichen Veröffentlichungen, von der ideologischen Zensur streng überwacht wurde. Der Bereich der Philosophie war auf besondere Weise betroffen. Denn während westliche Romane immer wieder übersetzt wurden, waren die Werke westlicher Philosophen und Philosophinnen des 20. Jahrhunderts nur aus den Kritiken an der so genannten "bürgerlichen Philosophie" bekannt oder aus Artikelübersetzungen, die $\mathrm{ab}$ und $\mathrm{zu}$ in den philosophischen Zeitschriften erschienen, begleitet von einem Kommentar aus "marxistischer" Sicht. Manche dieser Kommentare waren sehr ideologisch, andere wiederum versuchten die Zensur zu übertricksen, um den "westlichen" Autor sprechen zu lassen. Wenn man Heidegger, Sartre, Foucault und andere 
nichtmarxistische Gegenwartsphilosophen und -philosophinnen lesen wollte, musste man zum Original greifen. Als Assistentin an der Fakultät für Fremdsprachen an der Universität Sofia (1984 - 1986) sah ich deswegen eine meiner Aufgaben darin, durch die Übersetzung aus dem Französischen zu einer Öffnung der "anderen", der "westlichen" Kultur beizutragen, damit sich die Studierenden darüber selbst ein nicht stilisiertes Bild machen konnten. Während der Perestroika wurde es erstmals möglich einige Meister der Gegenwartsphilosophie, darunter Kierkegaard, Freud und Wittgenstein, ins Bulgarische zu übersetzen. Nach 1990 brach sogleich eine Lawine an Übersetzungen westlicher Philosophen und Philosophinnen aus. An dieser Arbeit war auch ich lange Zeit aktiv beteiligt, einerseits als Herausgeberin der Bücherreihen "Filosofi na XX vek" (Philosophen des 20. Jahrhunderts) und "Savremenna filosofia" (Philosophie der Gegenwart) bei zwei großen bulgarischen Verlagen, andererseits als Übersetzerin von Sartres und Ricœurs Werken, unter anderem von L'être et le néant und Le conflit des interprétations. Ich erwähne dies hier, weil ich der Meinung bin, dass man über Übersetzung nicht "philosophieren" kann, ohne selbst einmal ein Paar größere Übersetzungen gemacht zu haben.

Nun, meine innere Überzeugung während all dieser Jahre war und ist es immer noch, dass die Förderung von Fremdsprachenkenntnissen und die Übersetzungen eine wichtige Aufgabe ist. Sie ist notwendig, weil sie, erstens, dazu beiträgt die eigene Kultur und den eigenen Diskurs offen zu halten, anstatt ihn totalitär zu verschließen; zweitens, weil sie verhilft - wenn sie sachgemäß ausgeführt wird - die Mutterkultur zu bereichern und den eigenen Horizont zu erweitern; drittens, weil sie durch die Aufnahme des fremden Diskurses einen Austausch darüber ermöglicht und auch Instrumente liefert, sich ihm in einer kritischen Auseinandersetzung argumentierend entgegenzusetzen, anstatt sich durch Unwissen zu disqualifizieren. Dominanz kann weder durch Ignoranz noch durch Verbote überwunden werden. Die Herrschaft eines Diskurses kann nicht mit Gewalt durchbrochen werden, sondern nur durch einen anderen Diskurs, der ihm überlegen ist und es schafft diese Überlegenheit begrifflich geltend zu machen.

Wenn man die unglaubliche Menge an philosophischen und ganz allgemein an human- und sozialwissenschaftlichen Übersetzungen, die in den letzten 25 Jahren in "Osteuropa" erschienen sind in Betracht zieht, dann erscheinen sie zunächst als eine positive Öffnung. Dies war zwar eine verspätete, jedoch entscheidende Reaktion gegenüber dem jahrelangen dominanten und unglaubwürdig gewordenen totalitären Diskurs, der im Namen von Marx gesprochen, mit ihm aber oft wenig zu tun hatte. Es ist zum großen Teil 
durch diese Übersetzungsarbeit, dass die erstickende und monoton gewordene Ideologie, welche auf ihrer Unbeirrbarkeit beharrte, durch eine Vielfalt philosophischer Weltanschauungen ersetzt wurde. Diese Arbeit könnte jedoch, bei genauer Betrachtung, auch als ein problematischer und abenteuerlicher Lernprozess beschrieben werden, insofern viele misslungene Übersetzungen auf dem Markt erschienen und einen wesentlichen kulturellen Schaden angerichtet haben - zumindest aus meiner Sicht. Dieses Thema, das von den Sprachphilosophen bis jetzt übersehen wurde, könnte meines Erachtens ein Gegenstand interkulturellen Philosophierens werden, es geht hier aber weit über das uns hier interessierende Problem der Dominanz hinaus.

Wenn also die Übersetzungsarbeiten in den postkommunistischen Ländern zunächst als Reaktion auf eine langjährige ideologische Zensur und Unterdrückung interpretiert werden können, so wird durch eine genauere Betrachtung klar, dass durch diese sprachliche Gastfreundschaft die Türen für neue Dominanzverhältnisse geöffnet wurden, was ich im Folgenden an einigen Beispielen veranschaulichen möchte.

Ein großer Teil der übersetzten Bücher wurde durch Subventionen finanziert. Diese stammten aus den speziellen Programmen verschiedener Stiftungen wie dem "Fund for Central and East European Book Projects" der Europäischen Kulturstiftung, die Übersetzungsprogramme der "Ford Foundation" und der "Open Society Foundation", dem "Votosha"-Programm des Französischen Kulturministeriums, usw. Alle diese Programme unterstützten überwiegend Übersetzungen von Werken namenhafter Wissenschafterinnen und Wissenschaftler aus dem Westen in eine osteuropäische Sprache, aber nicht umgekehrt. ${ }^{4}$ Diese Subventionen waren Teil eines größeren, man könnte fast sagen globalen Projekts der Implementierung liberaler westlicher Ideologien in den postkommunistischen Ländern und wurden öffentlich als eine Art Beihilfe $\mathrm{zu}$ den begonnenen Demokratieprozessen dargestellt. Auf Initiative westlicher Institutionen wurden in den postkommunistischen Ländern verschiedene NGOs und Institute gegründet, neue Disziplinen wie die Women's Studies und Gender Studies eingerichtet und westliche

\footnotetext{
${ }^{4}$ Die Förderungspolitik bezog sich also auf Übersetzungen im Sinn von dem, was die Franzosen als "traduction" (Übersetzung von einer Fremdsprache in die Muttersprache) bezeichnen im Gegensatz zur Übersetzung als "version" (die umgekehrte Übersetzung von der Muttersprache in eine Fremdsprache). Die Differenz zwischen "traduction" und "version" scheint mir von Bedeutung gerade für koloniale, neokoloniale und postkoloniale Studien, weshalb ich sie später, bei der Beschreibung des Phänomens der Über-Setzung verwenden werde.
} 
Lehrkräfte, Berater und Experten entsandt, um zu unterrichten und Aufsicht zu führen. Dass viele Ost- und Westleute davon auch finanziell profitierten, daran besteht kein Zweifel (siehe Raynova 2010, 120-137). Dies führte zu Konflikten und man begann allmählich über eine "Kolonisation der Gesellschaftswissenschaften" zu sprechen. So wurde diesem Thema wurde eine spezielle Nummer der ungarischen Zeitschrift Replika gewidmet, in der die Einseitigkeiten des kulturellen "Ost-West-Austausches" angesprochen wurden (siehe Hadas, 1996 u. Csepeli 1996). Unter anderem die Tatsache, dass man an der Zentraleuropäischen Universität in Budapest von den Ostprofessorinnen und Ostprofessoren verlangte Englisch und andere westliche Sprachen zu beherrschen, sowie die westlichen Ideen, Geschichte und Kultur zu kennen, jedoch von den Professorinnen und Professoren aus dem Westen weder Sprachkenntnisse, noch andere Kenntnisse in Bezug auf Geschichte und Kultur des Gastgeberlandes gefordert wurden.

In verschiedenen Gesprächen und Publikationen berichteten Wissenschafterinnen, die sich vorwiegend mit Frauenforschung und Gender Studies beschäftigen, vom Mangel an Respekt seitens westlicher Kollegen, Kolleginnen und Institutionen. Die rumänische Philosophin Mihaela Miroiu teilte mir in einem Interview mit:

Due to the politics of translation, very few of our works were published in the West: Usually the financial support is focused on the reversal translation, according to an unwritten principle: 'The know how comes from West'. (Raynova 2010, 261).

Die ungarische Kollegin Maria Joó berichtet ihrerseits:

In Budapest gibt es ein Programm an der Zentraleuropäischen Universität - Program on Gender and Culture, - das die Möglichkeit bietet, einige GastprofessorInnen zu hören (...) Unsere Bemühungen wurden oft durch Unterstützung aus diesem Programm honoriert, etwa in Form von 'small grants', vom summer university courses etc. Bis jetzt war dieses Programm verständlicherweise amerikanisch orientiert. Die amerikanische Richtung erscheint manchmal wie eine Kolonisierung, die die Rezeption von feministischer Theorie in Osteuropa eher hindert als fördert. (ebd., 253)

In ihrem Artikel Real existierender Feminismus beschreibt die tschechische Soziologin Hana Havelková etwas Ähnliches:

Feministisch denkende Frauen, vorwiegend Amerikanerinnen (...) kamen, bewerteten und begannen zu belehren. Ich habe selbst Dutzende von Gesprächen erlebt (...), bei denen es immer um ein und das selbe ging: von ihrer Seite die Feststellung, dass unsere Gesellschaft sexistisch und patriarchalisch sei, die Frauen zweitrangige Bürger, außerdem konservativ, unfrei 
und diskriminiert usw.; von unserer Seite die Ablehnung dieser Diagnose in allen Punkten. (...) Vor allem haben wir diejenigen, die uns zu belehren suchten, darauf aufmerksam gemacht, dass sie unsere Wirklichkeit durch das Prisma ihrer eigenen Erfahrung und einer verinnerlichten feministischen Optik lesen. Das Resultat dieser mühseligen Diskussionen war Enttäuschung auf beiden Seiten: auf ihrer, weil wir uns nicht belehren lassen wollen, und auf unserer, weil sie die große Verschiedenheit unserer gelebten Erfahrung nicht respektieren, nicht fähig sind, ihr zu trauen und sie ernst zu nehmen. Der Vollständigkeit halber füge ich hinzu, dass keine gemeinsamen Sitzungen mehr stattfinden: Sie haben die ihren, und wir haben die unseren" (Havelková 1995, 147)5.

Die Jugoslawische Autorin Slavenka Drakulić fasst das Phänomen dieses Dominanzverhältnisses sehr treffend mit einem einzigen Satz: "The problem was that we could not let our American sisters tell us what to do in our countries, and that's exactly what they wanted to do". (Draculić 1998)

Doch die beschriebenen Probleme treffen nicht nur auf den Bereich der Frauenforschung und der Gender Studies zu, sondern auf den gesamten Bereich der Human- und Sozialwissenschaften, inklusive den Übersetzungs- und Austauschprogrammen. Die Frage, wieviele Werke "osteuropäischer" Wissenschaftler und Wissenschafterinnen ins Englische oder in andere westeuropäische Sprachen in den letzten 25 Jahren übersetzt wurden, ist nur ein Teil einer ganzen Reihe ähnlicher Fragen: Wie viele Gastprofessorinnen und Gastprofessoren aus den postkommunistischen Ländern wurden seit 1990 an westeuropäische Universitäten eingeladen? Wieviele hielten Hauptvorträge an Plenarsitzungen? In wieviele Projekte wurden sie miteinbezogen und zu welche Themen, ausgenommen solcher, die mit "Osteuropa" zu tun haben? In wievielen internationalen wissenschaftlichen Beiräten und Evaluierungskommissionen gibt es Professorinnen und Professoren aus Osteuropa?

Bei der Stellung dieser unbequemen Fragen kam es in der Vergangenheit oft zu Klagen und Anklagen. Förderlicher wäre jedoch ein reflexiver und selbstreflektierender Vorgang, um auf den Grund des Problems zu gehen. Womit haben wir es hier eigentlich zu tun? Mit einer Öffnung von "Ost" und einem Verschluss von "West"? Mit Hochmut, Vorurteilen und Ignoranz? Mit Mangel an Interesse bezüglich der wissenschaftlichen

${ }^{5}$ Siehe: Hana Havelková, "Real existierender Feminismus", in: Transit, Europäische Revue, Heft 9, Sommer 1995, S. 147. 
"Ostlandschaft"? Mit einem intellektuellen Eurozentrismus oder einem "westlichen" bzw. euro-amerikanischen "Universalismus", der den Rest der Welt als mehr oder weniger entlegene provinzielle Regionen behandelt und diese absichtlich an Distanz und in Abhängigkeit hält?

Ein Text von Julia Kristeva kann uns hier behilflich sein, um die Kulturdifferenz als Phänomen der geistigen Identität und Alterität, die zu dem Stigma der "rückständigen" Osteuropäer geführt hat, besser zu verstehen.

Bulgaren, [ihr, die ein] unsichtbarer, ungewollter, bleicher Fleck über die Klarheit [seid], düsterer Balkan, durchsetzt vom Mangel an Interesse des Okzidents, den ich verkörpere. (...) Obwohl ihr früh aufgebrochen seid, kommt ihr spät an, in einer veralteten Welt, die sich ständig erneuert und die die Zurückgebliebenen einfach nicht mag. (Kristeva 1995, 43) ${ }^{6}$

Obwohl Kristeva von den Bulgaren spricht, gilt dies in einem gewissen Sinne für alle "Osteuropäer". Diese "Zurückgebliebenen" (retardataires) - zurückgeblieben im "Orient" und in ihrer Entwicklung, zurückgeblieben in der Düsterkeit und der Finsternis des Denkens, beflecken die Klarheit der aufgeklärten Welt, in der für sie kein Platz ist. Sie sind zwar sehr früh aufgebrochen, haben es aber nicht geschafft voranzukommen, nun kommen sie nicht mit und bekommen nichts mit. Das werde, so Kristeva, gerade an den bulgarischen Übersetzungen sichtbar:

Man konstatierte, dass es an Wörtern fehlt und man hat in diese arme Sprache von empfindlichen Bauern und naiven Denkern ein Arsenal an geliehenen geschmacklosen und wurzellosen Ausdrücken hineingeschoben (ebd.) ${ }^{7}$

Die Übersetzungen sind also verfehlt, einerseits, weil die bulgarische Sprache eine "Bauernsprache" ist und, andererseits, weil die Bulgaren keinen Geschmack haben. "Faute de goût", meint Kristeva. Aber was ist der Geschmack, der den Rückständigen fehlt?

Der Geschmack ist die Rhetorik dieser Anerkennung, durch welche das Selbst, zentriert auf die Autorität, den Klan oder die individuelle Hypostase, dieselbe Sprache beim Anderen wie bei sich selbst entdeckt. Der Geschmack bestätigt die Differenz

6 "Bulgares, invisibles, indésirables, tache blanche sur la clarté, sombres Balkans transpercés par l'incuriosité de l'Occident dont je suis (...) Et bien que partis trop tôt, vous arrivez trop tard dans un monde trop vieux, mais qui ne cesse de se rajeunir et qui n'aime pas les retardataires.

7 "On a constaté que ça manquait de mots et on a fourré dans cette pauvre langue de paysans sensibles et de penseurs naïfs tout un arsenal d'emprunts sans saveurs ni racines". 
zwischen dem Selbst und dem Anderen, wenn und nur wenn diese Differenz harmonisiert wird; und so koste ich die Harmonie als einen aromatischen Geschmackstoff innerhalb der Autorität, des Klans und des Individuums, das die gleiche Sprache teilt. Der Geschmack ist höflich/geschliffen, weil er sich an die Seinigen richtet - an diese, welche dieselbe Autorität, denselben Klan, dieselben individuellen Werte teilen. ${ }^{8}$

Nun, Kristeva sagt es klar und eindeutig: Man hat die Werte der Autorität anzuerkennen, ihre Sprache zu sprechen und die eigenen Differenzen ihrem Diskurs anzupassen. Der Weg, auf den sich die Bulgaren (und manche andere) einst zu früh gemacht haben, führt nur in eine Richtung. Man nennt sie zwar "Europa", aber ihr eigentlicher Name ist "Okzident".

\section{Vom "Okzident" nach "Europa"}

Worin besteht nun das Phänomen des Missbrauchs der Gastfreundschaft der Übersetzung genauer? Welche Grundelemente und Grundstrukturen umfasst es?

In den sogenannten kolonialen und postkolonialen Studien wurde die Frage der Dominanz über die Sprache zwar thematisiert, jedoch weniger das Problem der Übersetzung. ${ }^{9}$ Der Grund dafür ist, dass man oft davon ausgegangen ist, dass der Kolonisator seine Sprache durchgesetzt hat, dass sie als Zweitsprache neben der Muttersprache oder sogar als Ersatz der Muttersprache instituiert wurde. Das stimmt zwar, aber das ist nicht alles. Wie der Kolonist, so setzt der herrschende Diskurs seine Sprache auf zweifache Art durch: Erstens, sie wird zur offiziellen, notwendig zu erlernenden Fremdsprache und zweitens, der Herrschende greift in die Muttersprache durch Übersetzungsarbeiten ein, damit alle, auch die, die seiner Sprache noch nicht mächtig sind, seine Autorität, seine Regeln und Werte, sowie seine Weltanschauung schnellstens kennenlernen und aufnehmen können. Da solche Übersetzungsarbeiten heute oft Hand an

8 "Le goût est la rhétorique de cette reconnaissance par laquelle le Moi, centré autour de l'Autorité, du clan ou de l'hypostase individuelle, retrouve chez l'autre le même langage que chez soi. Le goût affirme la différence du Moi et de l'autre si, et seulement si cette différence s'harmonise - et je goûte l'harmonie comme une saveur - au sein de l'Autorité, du clan, de l'individu partageant dès lors le même langage. Le goût est poli parce qu'il s'adresse aux siens : à ceux qui partagent la même autorité, le même clan, les mêmes valeurs individuelles".

${ }^{9}$ Unter den wenigen Forschungsarbeiten, die dieses Thema behandeln wäre die interessante Studie von Zauberga zu erwähnen (siehe z.B. Zauberga 2003). 
Hand mit den Informationsübermittlungen der großen Medien gehen, bleiben sie oft unbemerkt. Sie spielen jedoch eine grundlegende Rolle bei der Infiltrierung des dominanten Diskurses.

Was macht nun dieses Phänomen der Dominanz über die Sprache mittels der Übersetzung aus?

Die übliche Deutung, die man bei der Erklärung des Begriffs Übersetzung (Translation) gibt ist, dass sie eine Art Übertragung, Überlieferung von einer Sprache in die andere ist. Das ist insofern korrekt als die Übersetzung nicht unbedingt und nicht primär ein ethisches oder politisches Instrument darstellt, sondern ein Mittel der Verständigung, das oft zu ganz pragmatischen Zwecken verwendet wird, wie zum Beispiel um den Handel zwischen verschiedenen Völkern zu erleichtern. Um die Übersetzung in einem ganz bestimmten ethisch-politischen Sinn als Übertragung eines dominanten Diskurses zu erfassen, werde ich den Neologismus Über-Setzung als Arbeitsbegriff einführen. ÜberSetzung bedeutet in diesem Kontext ein über oder darüber Setzen, ein Überstülpen einer Sprache, eines Sprachspiels, eines Idioms oder einer Kultur über einer anderen durch Ausnutzung der Gastfreundschaft. Die dominante Sprache und ihr Diskurs greifen in die Muttersprache und ihre Kultur ein und setzen sich "auf" und "über" sie als etwas Wesentlicheres und universell Gültiges. Der dominante Diskurs, der sich durch die ÜberSetzung etablieren möchte, fordert Gehör, Verständnis und Akzeptanz und lehnt zugleich $\mathrm{ab}$, den Beherrschten zu hören und zu verstehen, da dieser nicht gleichwertig oder, um Kristeva zu paraphrasieren, nicht zu den "Seinigen", zu den Seinesgleichen gehört. Gerade auf dieser Ebene der Dialektik zwischen Aktivität und Passivität stößt man auf das ethischpolitische Paradox, auf das Sartre in seinen Spätschriften hingewiesen hat, nämlich: Mit dem Instituieren gewisser Normen und Werte wird vom Subjekt gefordert, dass es sie wählt und autonom in Handlungen umsetzt; zugleich aber werden ihm diese Wahlen und Handlungen aufgedrängt, sodass seine Autonomie beeinträchtigt wird. Vom Dominierten wird also eine gewisse Aktivität und Kreativität gefordert, seine Autonomie jedoch in Grenzen gehalten. Ricœurs Überlegungen über die sogenannte "traduction externe" und "traduction interne" hilft uns hier, das Problem auf dem Niveau des Verstehens zu verdeutlichen. Damit die Übersetzung des fremden Diskurses gelingt muss dieser internalisiert und Teil des inneren Lebens des Übersetzers werden. Der darüber-gesetzte Diskurs fordert also nicht nur eine adäquate Überlieferung, sondern auch eine interne Aufnahme und Annahme, ein Bekenntnis zu den Ideen und den Inhalten, die er propagiert. 
In einem hermeneutisch-phänomenologischen Sinn könnte man zusammengefasst sagen, dass das Phänomen der Über-Setzung als Herrschaft über und durch die Sprache folgende allgemeine Strukturen oder Merkmale aufweist:

1. Die Benutzung der sprachlichen Gastfreundschaft, um ein Dominanzverhältnis durch die Übersetzung zu etablieren, respektive die Verwendung der Übersetzung als Mittel zum Zweck, der die Herrschaft eines Diskurses über einen anderen ermöglicht.

2. Die Privilegierung einer Sprache, eines Sprachspiels oder eines Diskurses auf Kosten einer anderen Sprache oder eines anderen Diskurses, indem er nicht nur einen offiziellen Status bekommt, sondern auch sein Monopol als Hauptreferent standardisiert wird.

3. Die Einrichtung der Übersetzung als ideologische Institution der "traduction", das heißt als Übersetzung des Herrschenden Diskurses von der Fremdsprache in der Muttersprache des Beherrschten unter Ausschluss des umgekehrten Weges, der "version" (Übersetzung des Diskurses der Beherrschten in die Sprache der Herrschenden). Das Problem der Differenz zwischen "traduction" und "version"10, das hier im negativen Licht der Asymmetrie erscheint, wäre, positiv aufgefasst, von zentraler Bedeutung für die interkulturelle Kommunikation und der europäischen Integration, ist bis jetzt aber kaum thematisiert worden.

4. Die Verweigerung der diskursiven Reziprozität als Konsequenz der Ablehnung der Sprache und des Diskurses des Anderen als Beherrschten.

5. Die Dissimulierung der Ausnutzung der sprachlichen Freundschaft als Gabe oder als Gegengabe (z.B. durch einseitige Förderungsprogramme für Übersetzungen), um die Nicht-Reziprozität und die wahren Interessen zu verschleiern.

6. Die Praxis der Provinzialisierung als Festhaltung der Sprache und des Diskurses der Beherrschten an den Rand des dominierenden Diskurses in einer entlegenen "Provinz", aus der sie nur durch Beherrschung der Sprache und des Diskurses der Herrschenden eventuell ausbrechen und Gehör finden können.

Bezugnehmend auf die zu Beginn angeführte These von Domenico Jervolino, möchte ich nun den umgekehrten Schluss ziehen: Nein, Europa ist nicht "durch seine jahrhundertlange Geschichte voller Konflikte und Kriege gereift" und die europäische

\footnotetext{
${ }^{10}$ Siehe oben Fußnote 4.
} 
Sprache ist nicht die der Übersetzung. Zumindest noch nicht. Und sie wird es auch nicht werden, solange es keine Reziprozität zwischen Geben und Nehmen gibt.

\section{3. Übersetzung als translative Hermeneutik: Schlussfolgerungen}

Welche Schlussfolgerungen kann man aus der eidetischen Beschreibung der ÜberSetzung ziehen, bzw. welche philosophisch-hermeneutischen Instrumente könnten uns helfen Lösungsansetze für ein neues europäisches Übersetzungsethos zur Überwindung von sprachlichen und diskursiven Asymmetrien und Dominanzverhältnissen zu finden?

Ich habe an diversen philosophischen Veranstaltungen zwei entgegengesetzte Tendenzen beim Sprachgebrauch erlebt, die meines Erachtens beide gleichermaßen problematisch sind. Zum einen, eine extreme Anpassung an die englische Sprache durch Französischsprachige, die z.B. am Weltkongress für Philosophie in Boston 1998, an dem Französisch offizielle Sprache war, ausschließlich auf Englisch referiert haben und auch die Diskussionen in Englisch geführt haben wollten. Zum anderen, eine vehemente Ablehnung des Englischen und auch der deutschen Sprache von Spanischsprachigen, z.B. an einer internationalen Konferenz für interkulturelle Philosophie in Deutschland, an der eigentlich alle vier Sprachen - Deutsch, Spanisch, Englisch, Französisch - als gleichberechtigt gelten sollten. Nun, es ist verständlich, dass man von möglichst Vielen verstanden werden möchte und, dass man seine Texte in der Sprache verfassen, bzw. übersetzt haben will, in der auch der führende Diskurs zu den Problemen, die man behandelt, stattfindet. Das ist berechtigt und macht auch Sinn. Doch es bedeutet nicht, dass weil das Englische die neue lingua franca ist, nun auch jeder philosophische Diskurs auf Englisch stattfinden sollte. Kann man wirklich Spezialistin oder Spezialist in der Philosophie von Kant, Hegel oder Heidegger sein ohne ein Wort Deutsch zu können und in seinen Forschungen nur mit englischen Übersetzungen auskommen? Und umgekehrt, es wäre naiv zu glauben, dass man der Dominanz der englischen Sprache ein Ende setzen würde, wenn man Widerstand leistet, sie zu lernen oder zu sprechen. Deshalb wären hier zwei Dinge zu beachten. Erstens, dass man die dominanten Sprachen und Diskurse beherrschen sollte, wenn man sich nicht von ihnen blind beherrschen lassen möchte. Zweitens, dass authentische Kommunikation, die interkulturelle eingeschlossen, nur auf der Basis einer Balance zwischen Selbstachtung und Achtung der Gesprächspartner stattfinden kann. Wenn man sich und seine eigene Sprache und Kultur aufgibt, darf man nicht erwarten, dass Andere ein größeres Interesse daran finden. Zugleich sollte man den Anderen in seiner Sprache und Kultur auf dieselbe Art und 
Weise achten, auf die man selbst geachtet werden will. Ohne gegenseitige Wertschätzung kann es weder geistigen Austausch, noch wahre Gastfreundschaft geben. Es ist bedauerlich, dass vor dem Fall der Berliner Mauer es mehr Interesse an einen philosophischen OstWest-Austausch seitens des "Westens" gab, als jetzt im "Vereinten Europa" und vielleicht auch mehr Wertschätzung, da die Gegner als mehr oder weniger gleichwertig galten und das politische Interesse beider Seiten darin lag, den "Feind" für die eigene Ideologie zu gewinnen oder zu besiegen.

Die hermeneutische Phänomenologie als interkultureller Zugang könnte gerade hier einen wichtigen Beitrag leisten, indem sie uns Interpretationsinstrumente liefert, um bestehende Asymmetrien im Bereich der Übersetzung und der Gastfreundschaft aufzudecken und klarer zu artikulieren. Dieses Potential kann aber nur dann voll genutzt werden, wenn das interkulturelle Philosophieren in den postkommunistischen Ländern Fuß fasst, sodass postkoloniale Phänomene erkannt, analysiert und von den Betroffenen angesprochen werden können. Dies würde es ermöglichen die Kriterien der Förderungsprogramme kritisch zu überdenken, neu zu verhandeln und Wege vorzuschlagen, die ein Gleichgewicht des wissenschaftlichen Diskurses im neuen Europa ermöglichen würden. Die jetzt bestehenden Übersetzungsprogramme und Auslandsaufenthaltsförderungen sollten revidiert werden, um neue Möglichkeiten des Austausches zu schaffen, z.B. durch Programme für Übersetzungen von "osteuropäischen" Wissenschafterinnen und Wissenschaftler ins Deutsche, Französische und Englische, durch Gastprofessuren für Forschende und Lehrende aus den postkommunistischen Ländern und ihre Einbindung in internationale Beiräte und Gremien.

Die hermeneutische Phänomenologie als interkulturelles Philosophieren könnte nicht zuletzt einen Beitrag leisten, indem sie die Wege zu einer sprachlichen und diskursiven Vielfalt und einem reziproken europäischen Ost-West-Austausch konkret erörtert. Denn die kulturelle und wissenschaftliche Vielfalt Europas ist nicht etwas worauf man unmittelbar Zugriff hat, sondern es bedarf eines geschichtlichen, ideengeschichtlichen und polyphonen Zugangs, den ich in Anlehnung an Paul Ricœur und Leo Gabriel als eine translative Hermeneutik der europäischen Geschichte und Werte bezeichnen würde (siehe Raynova 2005, 110-128). Die Mannigfaltigkeit der europäischen Traditionen und Ideen zu erforschen und sie einem breiteren Publikum durch multilinguale Übersetzungen zugänglich zu machen wäre ein sine qua non für den Prozess der europäischen Integration, wenn diese nicht nur auf der Ebene der Ökonomie, der Politik und des Rechts stattfinden sollte. Die Aufgabe der Kritik, die ich hier anhand der Auslegung der "Über-Setzung" zu 
veranschaulichen versucht habe, ist in diesem Sinn ein positives Unterfangen, denn nur durch das Aufzeigen von Einseitigkeiten in der europäischen Sprach- und Kulturpolitik kann das Ricœursche Übersetzungsparadigma als eine reale Notwendigkeit und nicht nur als ideales Modell eines neuen Ethos für Europa wahrgenommen werden.

Prof. Dr. Yvanka B. Raynova, Institute for the Study of Societies and KnowledgeBulgarian Academy of Sciences, Sofia / Institut für Axiologische Forschungen, Wien,

raynova[at]iaf.ac.at

\section{Literaturangaben}

Csepeli, György et al. "Acquired Immune Deficiency Syndrome in Social Science in Eastern Europe," in Social Research, Vol 63, No 2, Summer 1996, 487-509.

Draculić, Slavenka. "What we Learned from Western Feminists," in Transitions, 15 January, 1998/1, 42-47 (online: http://www.tol.org/client/article/4983-what-we-learned-from-westernfeminists.html).

Hadas, Miklós (ed.). Colonization or Partnership? Eastern Europe and Western Social Sciences, a special issue of Replika: Hungarian Social Science Quarterly, 1996/1 (online: http://www.c3.hu/scripta/scripta0/replika/honlap/english/01/00contw.htm)

Havelková, Hana. "Real existierender Feminismus", in Transit, Europäische Revue, Heft 9, Sommer 1995, 146-158.

Jervolino, Domenico. "Übersetzung und hermeneutische Phänomenologie", in Labyrinth, Vol. 16, No. 1, 2014, .

Kristeva, Julia. "Bulgarie, ma souffrance", in L'Infini 51, automne 1995, 42-52.

Raynova, Yvanka. "Integrales denken im europäischen Kontext: Gabriel - Heidegger Ricœur", in Raynoa, Yvanka B./Moser, Susanne (Hrsg.). Das integrale und das gebrochene Ganze. Frankfurt am Main: Peter Lang, 2005, 91-128.

Raynova, Yvanka. Feministische Philosophie in europäischem Kontext. Genderdebatten zwischen "Ost" und "West". Wien/Köln/Weimar: Böhlau, 2010.

Ricœur, Paul. "Quel éthos nouveau pour l'Europe?", in Koslowski, Pierre (ed.) Imaginer l'Europe. Le marché intérieur européen, tâche culturelle et économique, Paris : Cerf, 1992, 107-116.

Ricœur, Paul. "Le paradigme de la traduction", in idem. Sur la traduction, Paris : Bayard, 2004, 42-43.

Zauberga, Ieva "Translation as Ideology-Driven Activity. Latvian Translation in the Soviet Period", in: Anu Mai Kõll, The Baltic Countries under Occupation. Soviet and Nazi Rule 1939-1991 (Acta Universitatis Stockholmiensis, Studia Baltica Stockholmiensia, 23), Stockholm: Stockholm University, 2003, 279-286. 